\title{
Optimization of Hybrid Manufacturing for Surface Quality, Material Consumption and Productivity Improvement
}

\author{
Damir Grguraš* - Davorin Kramar \\ University of Ljubljana, Faculty of Mechanical Engineering, Slovenia
}

This paper presents hybrid manufacturing process of 3D printing and milling. Fused deposition modeling (FDM) has been applied to improve product manufacturing performance, afterwards, milling was used to improve outer surface roughness. Today's FDM systems rely on the use of standard nozzle size with a diameter of $D_{1}=0.4 \mathrm{~mm}$. To achieve shorter production times a bigger nozzle size (diameter $D_{2}=1.1 \mathrm{~mm}$ ) has been used in this research. Optimization of technological parameters of hybrid manufacturing was carried out according to the minimal time of production, a minimal final surface roughness and minimal usage of material. In addition, these results were compared with optimal results obtained with the standard nozzle size. Significantly shorter production time, without affecting surface quality, was achieved when using bigger nozzle size.

Keywords: hybrid manufacturing, fused deposition modeling, milling, PLA material, design of experiments - DOE, empirical modeling and optimization, response surface methodology - RSM

Highlights

- Hybrid manufacturing of fused deposition modeling and milling is presented.

- $\quad$ Two different extruder nozzle sizes were used for fused deposition modeling.

- The optimization of technological parameters of hybrid manufacturing, according to the minimal production time, minimal surface roughness and minimal usage of material, has been carried out for both extruder nozzle sizes.

- Optimal technological parameters obtained with standard and nonstandard extruder nozzle size are revealed by comparison.

\section{INTRODUCTION}

Fused deposition modeling (FDM) is an additive manufacturing technology commonly used for modeling, prototyping, and production applications. Due to the relatively low manufacturing cost, it is one of the most commonly used 3D printing technique. Nevertheless, dimensional accuracy, surface roughness and manufacturing times are highly dependent on the process parameters. In some cases, FDM products do not meet desirable dimensional accuracy and surface roughness despite the wellselected process parameters. Thereby, in order to improve product quality, hybrid manufacturing with additional machining can be applied. Thus, FDM process can be applied for product creation, while machining, e.g. milling can be used to improve dimensional accuracy and overall surface roughness. The aim of applying both selected technologies (FDM and milling) in combination is to remove drawbacks of individual one. The milling process has its limitations in the type of geometry where close surface production is required, since the cutting tool by itself cannot produce them. On the other hand, FDM technology almost does not pose any limitations regarding generating complex geometry shapes, but lacks in produced surface quality. These drawbacks can be minimized with the use of both technologies in combination [1] and [2].

Few studies can be found, where additive and subtractive technologies are combined, while most of them present studies of metal parts processing. In the work of Song et al. [3] and [4], 3-axis CNC milling centre was upgraded with two welding guns vertically attached to the spindle housing. For parts creation the welding process gas metal arc welding (GMAW) has been applied followed by significant improvement of dimensional accuracy by machining. In the Norway - Slovenian collaborative research [5], new working hybrid cell for hybrid manufacturing has been developed. A commercial machine for additive manufacturing has been combined with a machining centre into one working cell and both processes were integrated into one unified controlled system. The position accuracy has been ensured with the usage of fixtures on standard pallets and therefore the dimensional accuracy of the products improved. Furthermore, Yamazaki [6] developed hybrid multi - tasking machine tool by equipping laser metal deposition functionality in addition to existing integrated turning and milling capabilities. This machine tool concept enables a further evolution of done-in-one processes enabling building nearnet shape components to be produced by additive manufacturing and then quickly generating the 
net shape through high-precision finish machining operations. Lee et al. [7] developed hybrid rapid prototyping system using FDM and 5-axis machining. One of the innovative features of the system involved installing the cutter spindle on one end and the FDM extruder on the other end of the rotary B axis. Thus, allowing the machine to switch between the two activities without any extra actuation system, thereby simplifying the mechanism complexity and reducing the time to find the position of the cutter relative to the FDM part for subsequent machining. Having five axis on this machine resulted in several benefits, e.g. five-axis machine could make the overhang feature without using the support material. All of these studies are showing current trend in upgrading existing machine tools or developing completely new hybrid machine tools for combination of additive and subtractive machining. Additionally, few studies were made for FDM process parameters optimization for better dimensional accuracy. Mohamed et al. [8] used l-optimality criterion for the optimization of FDM process parameters in order to address the limitations of the commonly used traditional designs. In the same study mathematical models of nonlinear relationships between process parameters and dimensional accuracy were also developed. Kaveh et al. [9] presented a newly developed experimental method to determine the optimum quantity of each effective printing parameters for high impact polystyrene material. Rao and Rai [10] used teaching-learning-based optimization (TLBO) algorithm and non-dominated Sorting TLBO for FDM optimization procedure. Further, in study [11], multi objective optimization was used for sustainable manufacturing. Threedimensional statistical approach for determining the manufacturing tolerances has been used in study [12] In this study, in order to achieve optimal technological parameters settings of hybrid manufacturing, i.e. 3D printing and milling parameters, a response surface methodology has been applied.

\section{PROCESS PRINCIPLE AND EXPERIMENTAL SETUP OF 3D PRINTING AND MILLING}

The CNC machine for hybrid manufacturing has been developed for this research. It combines a 3D printing and milling system, as shown in Fig. 1. The 3D printing system relies on FDM technique where the plastic filament is led to the heating body and is afterwards deposited through the nozzle down onto a build platform. The milling system consists of a Kress milling motor with nominal rated input power of 800 W. The spindle speed can be set between 10.000 to
$29.000 \mathrm{rpm}$. The specimens, i. e. cubes (dimensions $22 \mathrm{~mm} \times 22 \mathrm{~mm} \times 22 \mathrm{~mm}$ ) from PLA material, were manufactured by the FDM process. Afterwards, milling process has been applied in order to achieve better surface roughness.

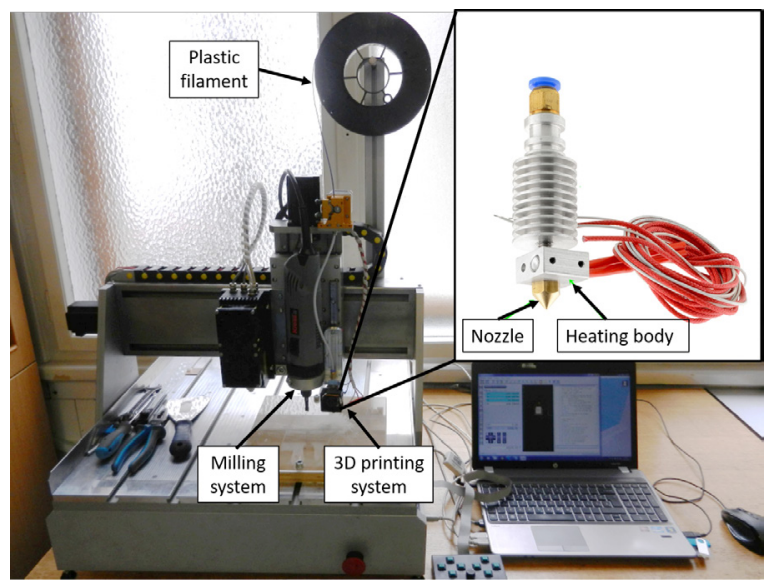

Fig. 1. Machine for hybrid manufacturing consists of $3 D$ printing (FDM) and milling systems [1]

\section{EXPERIMENTAL APPROACH AND RESULTS}

\subsection{Technological Parameters of Hybrid Manufacturing}

The following technological parameters, which were determined based on the previous knowledge and preliminary experiments of hybrid manufacturing, were chosen:

- $\quad$ spindle speed of milling tool $n\left[\mathrm{~min}^{-1}\right]$,

- layer height $h[\mathrm{~mm}]$,

- material compensation flow $\Phi[\%]$,

- $\quad$ printing speed $v[\mathrm{~mm} / \mathrm{s}]$,

- feed speed of milling tool $v_{f}[\mathrm{~mm} / \mathrm{min}]$,

- $\quad$ milling depth $a_{p}$ [mm].

For hybrid manufacturing two different nozzle sizes were used; standard (diameter, $D_{1}=0.4 \mathrm{~mm}$ ) and nonstandard size (diameter, $D_{2}=1.1 \mathrm{~mm}$ ). The limit values (minimal and maximal values) of the technological parameters were determined based on pilot experiments and with the integration of proposed values of a $3 \mathrm{D}$ printing control software CURA.

\subsection{Design and Execution of Experiments}

Because, technological parameters for nozzle sizes $D_{1}=0.4 \mathrm{~mm}$ and $D_{2}=1.1 \mathrm{~mm}$ differ, two separate experiment designs had to be created. Each technological parameter was examined on three levels. For the level -1 the minimum values of the parameters were considered, and for the level +1 the maximum 
values. Values of a mid-level 0 represent the average between levels -1 and +1 . For both nozzles, the parameter values at each level are shown in the Tables 1 and 2.

Table 1. Values of technological parameters at each level for nozzle size $D_{1}=0.4 \mathrm{~mm}$

\begin{tabular}{ccccccc}
\hline & \multicolumn{6}{c}{ Technological parameter } \\
\cline { 2 - 7 } Level & $\begin{array}{c}n \\
{\left[\mathrm{~min}^{-1}\right]}\end{array}$ & $\begin{array}{c}h \\
{[\mathrm{~mm}]}\end{array}$ & $\begin{array}{c}\Phi \\
{[\%]}\end{array}$ & $\begin{array}{c}v \\
{[\mathrm{~mm} / \mathrm{s}]}\end{array}$ & $\begin{array}{c}v_{f} \\
{[\mathrm{~mm} / \mathrm{min}]}\end{array}$ & $\begin{array}{c}a_{p} \\
{[\mathrm{~mm}]}\end{array}$ \\
\hline-1 & 10000 & 0.10 & 55.0 & 15 & 200 & 0.10 \\
\hline 0 & 15500 & 0.20 & 67.5 & 40 & 400 & 0.20 \\
\hline+1 & 21000 & 0.30 & 80.0 & 65 & 600 & 0.30 \\
\hline
\end{tabular}

Table 2. Values of technological parameters at each level for nozzle size $D_{2}=1.1 \mathrm{~mm}$

\begin{tabular}{ccccccc}
\hline & \multicolumn{6}{c}{ Technological parameter } \\
\cline { 2 - 7 } Level & $\begin{array}{c}n \\
{\left[\mathrm{~min}^{-1}\right]}\end{array}$ & $\begin{array}{c}h \\
{[\mathrm{~mm}]}\end{array}$ & $\begin{array}{c}\Phi \\
{[\%]}\end{array}$ & $\begin{array}{c}v \\
{[\mathrm{~mm} / \mathrm{s}]}\end{array}$ & $\begin{array}{c}v_{f} \\
{[\mathrm{~mm} / \mathrm{min}]}\end{array}$ & $\begin{array}{c}a_{p} \\
{[\mathrm{~mm}]}\end{array}$ \\
\hline-1 & 10000 & 0.30 & 60.0 & 10 & 200 & 0.41 \\
\hline 0 & 15500 & 0.55 & 70.5 & 25 & 400 & 0.55 \\
\hline+1 & 21000 & 0.80 & 80.0 & 40 & 600 & 0.69 \\
\hline
\end{tabular}

For the design of experiments (DOE) with 6 technological parameters on three levels, Taguchi orthogonal array $\mathrm{L}_{27}$ has been applied and two parallel DOEs for both nozzle sizes were created using Design-Expert software. The DOEs for both nozzle sizes are presented in the next Chapter 2.3 in Tables 4 and 5 (left of the thick line).

Table 3. List of output parameters

\begin{tabular}{ccl}
\hline $\begin{array}{c}\text { Output } \\
\text { parameter }\end{array}$ & Unit & \multicolumn{1}{c}{ Parameter description } \\
\hline$R a_{h}$ & {$[\mu \mathrm{m}]$} & $\begin{array}{l}\text { Arithmetic deviation profile in the direction } \\
\text { perpendicularly to the loading material }\end{array}$ \\
\hline$R y_{h}$ & {$[\mu \mathrm{m}]$} & $\begin{array}{l}\text { Maximal surface roughness in the direction } \\
\text { perpendicularly to the loading material }\end{array}$ \\
\hline$R a_{l}$ & {$[\mu \mathrm{m}]$} & $\begin{array}{l}\text { Arithmetic deviation profile in the direction of } \\
\text { loading material }\end{array}$ \\
\hline$R y_{l}$ & {$[\mu \mathrm{m}]$} & $\begin{array}{l}\text { Maximal surface roughness in the direction of } \\
\text { loading material }\end{array}$ \\
\hline$M D$ & {$[\mathrm{~m}]$} & $\begin{array}{l}\text { Material deposition - used material for FDM } \\
\text { process }\end{array}$ \\
\hline$t$ & {$[\mathrm{~s}]$} & \begin{tabular}{l} 
Time for hybrid manufacturing \\
\hline
\end{tabular}
\end{tabular}

The output parameters, which are listed and described in Table 3, represent responses of hybrid

Table 4. Design of experiments and corresponding results; nozzle size $D_{1}=0.4 \mathrm{~mm}$

\begin{tabular}{|c|c|c|c|c|c|c|c|c|c|c|c|c|}
\hline & $\begin{array}{c}n \\
{\left[\mathrm{~min}^{-1}\right]}\end{array}$ & $\begin{array}{c}h \\
{[\mathrm{~mm}]}\end{array}$ & $\begin{array}{c}\Phi \\
{[\%]}\end{array}$ & $\begin{array}{c}v \\
{[\mathrm{~mm} / \mathrm{s}]}\end{array}$ & $\begin{array}{c}v_{f} \\
{[\mathrm{~mm} / \mathrm{min}]}\end{array}$ & $\begin{array}{c}a_{p} \\
{[\mathrm{~mm}]}\end{array}$ & $\begin{array}{l}R a_{h} \\
{[\mu \mathrm{m}]}\end{array}$ & $\begin{array}{l}R y_{h} \\
{[\mu \mathrm{m}]}\end{array}$ & $\begin{array}{l}R a_{l} \\
{[\mu \mathrm{m}]}\end{array}$ & $\begin{array}{l}R y_{l} \\
{[\mu \mathrm{m}]}\end{array}$ & $\begin{array}{c}M D \\
{[\mathrm{~m}]}\end{array}$ & $\begin{array}{c}t \\
{[\mathrm{~s}]}\end{array}$ \\
\hline 1 & 10000 & 0.10 & 55.0 & 15 & 200 & 0.10 & 5.69 & 47.35 & 4.97 & 44.15 & 1.02 & 8066 \\
\hline 2 & 10000 & 0.10 & 67.5 & 40 & 400 & 0.20 & 5.05 & 42.40 & 6.57 & 48.02 & 1.21 & 7324 \\
\hline 3 & 10000 & 0.10 & 80.0 & 65 & 600 & 0.30 & 5.86 & 45.63 & 6.62 & 50.40 & 1.48 & 7191 \\
\hline 4 & 10000 & 0.20 & 55.0 & 40 & 400 & 0.30 & 3.89 & 45.11 & 5.85 & 47.16 & 1.02 & 3715 \\
\hline 5 & 10000 & 0.20 & 67.5 & 65 & 600 & 0.10 & 2.43 & 22.18 & 4.19 & 37.27 & 1.24 & 3647 \\
\hline 6 & 10000 & 0.20 & 80.0 & 15 & 200 & 0.20 & 5.55 & 40.55 & 5.71 & 43.97 & 1.48 & 4100 \\
\hline 7 & 10000 & 0.30 & 55.0 & 65 & 600 & 0.20 & 1.93 & 25.18 & 2.39 & 23.58 & 1.01 & 2477 \\
\hline 8 & 10000 & 0.30 & 67.5 & 15 & 200 & 0.30 & 5.82 & 41.24 & 6.28 & 44.95 & 1.24 & 2776 \\
\hline 9 & 10000 & 0.30 & 80.0 & 40 & 400 & 0.10 & 4.71 & 50.47 & 5.50 & 38.99 & 1.48 & 2522 \\
\hline 10 & 15500 & 0.10 & 55.0 & 40 & 600 & 0.20 & 5.45 & 48.25 & 5.68 & 46.61 & 1.02 & 7313 \\
\hline 11 & 15500 & 0.10 & 67.5 & 65 & 200 & 0.30 & 3.53 & 27.65 & 4.05 & 29.91 & 1.24 & 7210 \\
\hline 12 & 15500 & 0.10 & 80.0 & 15 & 400 & 0.10 & 5.33 & 45.29 & 5.80 & 41.53 & 1.48 & 8053 \\
\hline 13 & 15500 & 0.20 & 55.0 & 65 & 200 & 0.10 & 7.96 & 82.49 & 5.62 & 48.47 & 1.02 & 3668 \\
\hline 14 & 15500 & 0.20 & 67.5 & 15 & 400 & 0.20 & 5.68 & 45.58 & 5.47 & 41.10 & 1.24 & 4082 \\
\hline 15 & 15500 & 0.20 & 80.0 & 40 & 600 & 0.30 & 6.06 & 46.26 & 6.69 & 52.11 & 1.48 & 3714 \\
\hline 16 & 15500 & 0.30 & 55.0 & 15 & 400 & 0.30 & 6.13 & 50.70 & 6.62 & 47.77 & 1.01 & 2761 \\
\hline 17 & 15500 & 0.30 & 67.5 & 40 & 600 & 0.10 & 5.29 & 42.10 & 5.54 & 45.38 & 1.24 & 2516 \\
\hline 18 & 15500 & 0.30 & 80.0 & 65 & 200 & 0.20 & 5.37 & 41.39 & 5.30 & 40.88 & 1.48 & 2496 \\
\hline 19 & 21000 & 0.10 & 55.0 & 65 & 400 & 0.30 & 5.29 & 42.82 & 5.71 & 44.66 & 1.02 & 7202 \\
\hline 20 & 21000 & 0.10 & 67.5 & 15 & 600 & 0.10 & 6.71 & 48.43 & 6.33 & 45.22 & 1.24 & 8043 \\
\hline 21 & 21000 & 0.10 & 80.0 & 40 & 200 & 0.20 & 2.88 & 26.48 & 2.67 & 19.78 & 148 & 7337 \\
\hline 22 & 21000 & 0.20 & 55.0 & 15 & 600 & 0.20 & 6.20 & 48.38 & 5.76 & 45.69 & 1.02 & 4079 \\
\hline 23 & 21000 & 0.20 & 67.5 & 40 & 200 & 0.30 & 5.37 & 52.67 & 5.58 & 47.86 & 1.24 & 3736 \\
\hline 24 & 21000 & 0.20 & 80.0 & 65 & 400 & 0.10 & 5.14 & 47.09 & 5.44 & 43.72 & 1.48 & 3657 \\
\hline 25 & 21000 & 0.30 & 55.0 & 40 & 200 & 0.10 & 6.56 & 70.75 & 5.61 & 49.68 & 1.01 & 2540 \\
\hline 26 & 21000 & 0.30 & 67.5 & 65 & 400 & 0.20 & 6.26 & 52.78 & 5.52 & 48.43 & 1.23 & 2482 \\
\hline 27 & 21000 & 0.30 & 80.0 & 15 & 600 & 0.30 & 6.27 & 47.76 & 6.93 & 53.11 & 1.48 & 2759 \\
\hline
\end{tabular}


Table 5. Design of experiments and corresponding results; nozzle size $D_{2}=1.1 \mathrm{~mm}$

\begin{tabular}{|c|c|c|c|c|c|c|c|c|c|c|c|c|}
\hline & $\begin{array}{c}n \\
{\left[\mathrm{~min}^{-1}\right]}\end{array}$ & $\begin{array}{c}h \\
{[\mathrm{~mm}]}\end{array}$ & $\begin{array}{c}\Phi \\
{[\%]}\end{array}$ & $\begin{array}{c}v \\
{[\mathrm{~mm} / \mathrm{s}]}\end{array}$ & $\begin{array}{c}v_{f} \\
{[\mathrm{~mm} / \mathrm{min}]}\end{array}$ & $\begin{array}{c}a_{p} \\
{[\mathrm{~mm}]}\end{array}$ & $\begin{array}{l}R a_{h} \\
{[\mu \mathrm{m}]}\end{array}$ & $\begin{array}{l}R y_{h} \\
{[\mu \mathrm{m}]}\end{array}$ & $\begin{array}{l}R a_{l} \\
{[\mu \mathrm{m}]}\end{array}$ & $\begin{array}{l}R y_{l} \\
{[\mu \mathrm{m}]}\end{array}$ & $\begin{array}{c}M D \\
{[\mathrm{~m}]}\end{array}$ & $\begin{array}{c}t \\
{[\mathrm{~s}]}\end{array}$ \\
\hline 1 & 10000 & 0.30 & 60.0 & 10 & 200 & 0.41 & 5.10 & 47.26 & 4.91 & 41.65 & 2.09 & 2330 \\
\hline 2 & 10000 & 0.30 & 70.0 & 25 & 400 & 0.55 & 5.64 & 47.73 & 6.92 & 52.15 & 2.43 & 1977 \\
\hline 3 & 10000 & 0.30 & 80.0 & 40 & 600 & 0.69 & 4.83 & 45.01 & 5.82 & 45.32 & 2.78 & 1901 \\
\hline 4 & 10000 & 0.55 & 60.0 & 25 & 400 & 0.69 & 4.81 & 49.14 & 6.26 & 50.37 & 2.05 & 1102 \\
\hline 5 & 10000 & 0.55 & 70.0 & 40 & 600 & 0.41 & 9.19 & 114.12 & 4.33 & 43.31 & 2.39 & 1061 \\
\hline 6 & 10000 & 0.55 & 80.0 & 10 & 200 & 0.55 & 16.08 & 162.03 & 10.90 & 73.92 & 2.73 & 1312 \\
\hline 7 & 10000 & 0.80 & 60.0 & 40 & 600 & 0.55 & 1.18 & 8.44 & 3.75 & 27.50 & 2.04 & 721 \\
\hline 8 & 10000 & 0.80 & 70.0 & 10 & 200 & 0.69 & 2.24 & 17.52 & 3.64 & 24.02 & 2.38 & 900 \\
\hline 9 & 10000 & 0.80 & 80.0 & 25 & 400 & 0.41 & 2.57 & 18.40 & 3.57 & 21.06 & 2.72 & 763 \\
\hline 10 & 15500 & 0.30 & 60.0 & 25 & 600 & 0.55 & 4.30 & 33.67 & 4.43 & 33.19 & 2.09 & 1973 \\
\hline 11 & 15500 & 0.30 & 70.0 & 40 & 200 & 0.69 & 24.75 & 198.15 & 9.61 & 75.19 & 2.43 & 1921 \\
\hline 12 & 15500 & 0.30 & 80.0 & 10 & 400 & 0.41 & 7.47 & 61.69 & 8.65 & 57.98 & 2.78 & 2319 \\
\hline 13 & 15500 & 0.55 & 60.0 & 40 & 200 & 0.41 & 14.65 & 171.67 & 5.64 & 40.99 & 2.05 & 1079 \\
\hline 14 & 15500 & 0.55 & 70.0 & 10 & 400 & 0.55 & 7.56 & 55.75 & 8.94 & 60.33 & 2.39 & 1293 \\
\hline 15 & 15500 & 0.55 & 80.0 & 25 & 600 & 0.69 & 9.07 & 120.38 & 6.09 & 54.23 & 2.73 & 1103 \\
\hline 16 & 15500 & 0.80 & 60.0 & 10 & 400 & 0.69 & 2.97 & 26.14 & 5.25 & 38.50 & 2.04 & 882 \\
\hline 17 & 15500 & 0.80 & 70.0 & 25 & 600 & 0.41 & 3.64 & 31.49 & 4.07 & 34.31 & 2.38 & 752 \\
\hline 18 & 15500 & 0.80 & 80.0 & 40 & 200 & 0.55 & 8.98 & 65.07 & 8.11 & 60.07 & 2.72 & 752 \\
\hline 19 & 21000 & 0.30 & 60.0 & 40 & 400 & 0.69 & 10.63 & 158.18 & 6.20 & 42.14 & 2.09 & 1907 \\
\hline 20 & 21000 & 0.30 & 70.0 & 10 & 600 & 0.41 & 7.76 & 56.27 & 6.78 & 48.55 & 2.43 & 2311 \\
\hline 21 & 21000 & 0.30 & 80.0 & 25 & 200 & 0.55 & 8.09 & 55.75 & 9.48 & 68.24 & 2.78 & 1989 \\
\hline 22 & 21000 & 0.55 & 60.0 & 10 & 600 & 0.55 & 5.73 & 42.42 & 7.87 & 53.77 & 2.05 & 1282 \\
\hline 23 & 21000 & 0.55 & 70.0 & 25 & 200 & 0.69 & 25.00 & 154.25 & 11.12 & 95.37 & 2.39 & 1121 \\
\hline 24 & 21000 & 0.55 & 80.0 & 40 & 400 & 0.41 & 6.30 & 54.83 & 6.22 & 49.46 & 2.73 & 1068 \\
\hline 25 & 21000 & 0.80 & 60.0 & 25 & 200 & 0.41 & 9.60 & 98.97 & 8.04 & 61.24 & 2.04 & 771 \\
\hline 26 & 21000 & 0.80 & 70.0 & 40 & 400 & 0.55 & 3.40 & 43.69 & 7.06 & 48.81 & 2.38 & 730 \\
\hline 27 & 21000 & 0.80 & 80.0 & 10 & 600 & 0.69 & 6.82 & 51.13 & 5.39 & 42.63 & 2.72 & 888 \\
\hline
\end{tabular}

manufacturing and were followed and measured for each experiment set. Surface roughness of the machined parts was measured with contact surface roughness measuring device Mitutoyo Surftest SJ 301.

\subsection{Evaluation and Analysis of Result}

In Tables 4 and 5 (right of the thick line) the results for both nozzle sizes $\left(D_{1}=0.4 \mathrm{~mm}\right.$ and $\left.D_{2}=1.1 \mathrm{~mm}\right)$ conducted after carrying out all of the 27 experiments are given. This results were further used to analyse the influence of the technological parameters on the output responses as well as to obtain mathematical models for their final optimisation.

The mathematical models were obtained and evaluated by deployment of a computer program Design-Expert, which develops and analyses regression models using analysis of variance (ANOVA). Those acquired models have been evaluated on the basis of $F$-value, $p$-value, $R^{2}$, adjusted $R^{2}\left(\operatorname{Adj}-R^{2}\right)$, predicted $R^{2}\left(\right.$ Pred $\left.-R^{2}\right)$ and $S / N$ ratio (signal to noise ratio). Please see Table 6 and 7 .

\subsection{Regression Models and Their Interpretation}

In the following chapter, the regression models for the different responses and both nozzle sizes are presented through mathematical equations as well as 3D response surface graphs.

Regression models for roughness $R a_{h}$ :

- $\quad$ Nozzle size $D_{1}=0.4 \mathrm{~mm}$ :

$$
\begin{aligned}
& R a_{h}=12.07961+3.2641 \cdot 10^{-4} \cdot n+ \\
& 18.38333 \cdot h-0.15542 \cdot \phi-0.030158 \cdot v_{f}+ \\
& 8.49242 \cdot 10^{-7} \cdot n \cdot v_{f}-0.042417 \cdot h \cdot v_{f}+ \\
& 3.67 \cdot 10^{-4} \cdot \phi \cdot v_{f}-1.83104 \cdot 10^{-8} \cdot n^{2} .
\end{aligned}
$$

- $\quad$ Nozzle size $D_{2}=1.1 \mathrm{~mm}$ :

$$
\begin{aligned}
& \ln \left(R a_{h}\right)=0.54592+8.93868 \cdot h+0.056732 \cdot v- \\
& 5.08715 \cdot 10^{-3} \cdot v_{f}-1.28640 \cdot 10^{-4} \cdot v \cdot v_{f}- \\
& 9.38239 \cdot h^{2}+8.21082 \cdot 10^{-6} \cdot v_{f}^{2} .
\end{aligned}
$$

The main influence on surface roughness in the direction perpendicular to the loading material flow, for both nozzle sizes, has layer height $h$ and feed speed 
Table 6. Evaluation of regression models; nozzle size $D_{1}=0.4 \mathrm{~mm}$

\begin{tabular}{ccccccccc}
\hline $\begin{array}{c}\text { Output } \\
\text { parameter }\end{array}$ & $F$-value & $p$-value & Regresor-influential parameter & $\begin{array}{c}\text { Atypical hierarchical } \\
\text { parameter }\end{array}$ & $R^{2}$ & Adj- $R^{2}$ & Pred- $R^{2}$ & $S / N$ \\
\hline$R a_{h}$ & 9.03 & $<0.0001$ & $n, n \times v_{f}, h \times v_{f}, \phi \times v_{f}$ & $n_{2}, h, \phi, v_{f}$ & 0.801 & 0.712 & 0.5379 & 12.48 \\
\hline$R y_{h}$ & 5.83 & 0.0011 & $*$ & $*$ & 0.811 & 0.672 & 0.164 & 10.88 \\
\hline$R a_{l}$ & 8.99 & $<0.0001$ & $a_{p}, n \times v_{f}, h \times v_{f}, \phi \times v_{f}, v_{f 2}$ & $n, h, \phi, v_{f}, n \times h$ & 0.849 & 0.755 & 0.597 & 12.28 \\
\hline$R y_{l}$ & 12.27 & $<0.0001$ & $n, v_{f}, a_{p}, n \times h, n \times v_{f}, h \times v_{f}, \phi \times v_{f}, v_{2}$ & $h, \phi, v, \phi \times v, h_{2}$ & 0.925 & 0.849 & 0.668 & 15.04 \\
\hline$M D$ & 39259.52 & $<0.0001$ & $\phi$ & $/$ & 0.999 & 0.999 & 0.999 & 343.19 \\
\hline$t$ & 3121.86 & $<0.0001$ & $h, v$ & $/$ & 0.996 & 0.996 & 0.995 & 123.40 \\
\hline
\end{tabular}

* Useless model because Adj- $R^{2}$ and Pred- $R^{2}$ differ more than 0.2.

Table 7. Evaluation of regression models for hybrid; nozzle size $D_{2}=1.1 \mathrm{~mm}$

\begin{tabular}{|c|c|c|c|c|c|c|c|c|}
\hline $\begin{array}{c}\text { Output } \\
\text { parameter }\end{array}$ & $F$-value & $p$-value & Regresor-influential parameter & $\begin{array}{c}\text { Atypical } \\
\text { hierarchical } \\
\text { parameter }\end{array}$ & $R^{2}$ & $\operatorname{Adj}-R^{2}$ & Pred- $R^{2}$ & $S / N$ \\
\hline$R a_{h}$ & 7.97 & 0.0002 & $h, v_{f}, v \times v_{f}, h^{2}$ & $v$ & 0.705 & 0.617 & 0.427 & 10.90 \\
\hline$R y_{h}$ & 5.90 & 0.0012 & * & * & 0.835 & 0.693 & 0.344 & 9.95 \\
\hline$R a_{l}$ & 12.50 & $<0.0001$ & $n, \phi, v_{f}, v \times a_{p}, h^{2}, a_{p}^{2}$ & $h, v$ & 0.902 & 0.830 & 0.669 & 12.11 \\
\hline$R y_{l}$ & 14.40 & $<0.0001$ & $n, \phi, v_{f}, a_{p}, n \times h, n \times v, \phi \times v_{f}, v \times a_{p}, v_{f} \times a_{p}, h^{2}$ & $h, v$ & 0.935 & 0.870 & 0.673 & 15.68 \\
\hline$M D$ & 246000 & $<0.0001$ & $h, \phi, h \times \phi, h^{2}, \phi^{2}$ & 1 & 1.000 & 1.000 & 1.000 & 1190.27 \\
\hline$t$ & 1157.56 & $<0.0001$ & $h, v$ & I & 0.990 & 0.989 & 0.987 & 80.32 \\
\hline
\end{tabular}

*Useless model because Adj- $R^{2}$ and Pred- $R^{2}$ differ more than 0.2.

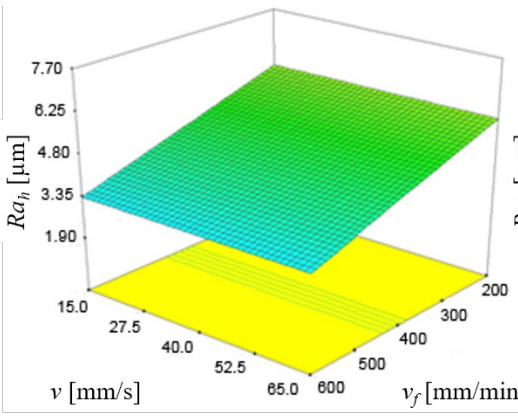

a)

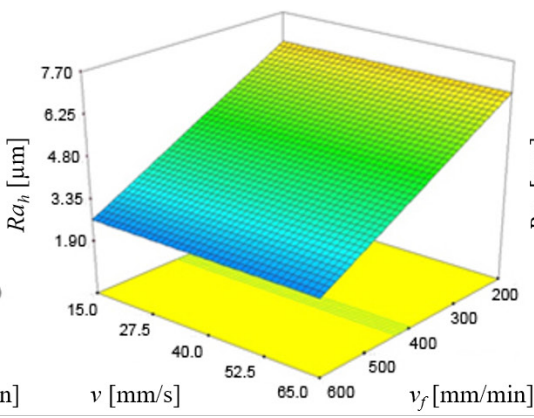

b)

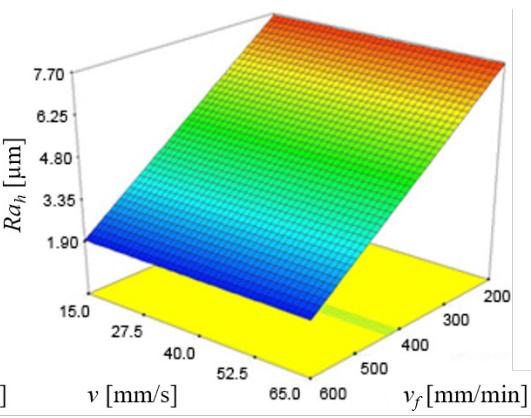

c)

Fig. 2. Influence of printing speed $v$ and feed speed of milling tool $v_{f}$ on surface roughness $R a_{h}$ by different layer heights $h$ using nozzle size $D_{1}=0.4 \mathrm{~mm}, n=10000 \mathrm{~min}^{-1}, \Phi 55 \%, a_{p}=0.1 \mathrm{~mm}$; a) $\left.h=0.1 \mathrm{~mm}, b\right) h=0.2 \mathrm{~mm}$, and c) $h=0.3 \mathrm{~mm}$

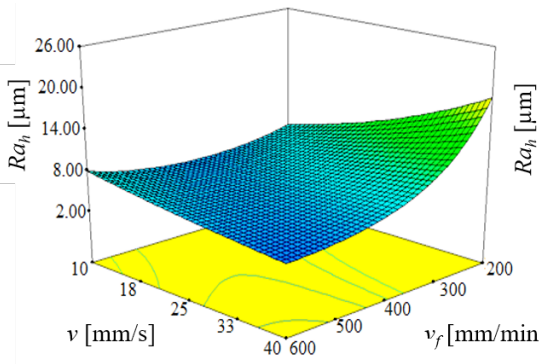

a)

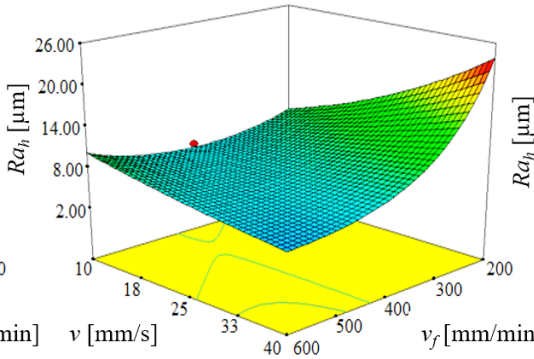

b)

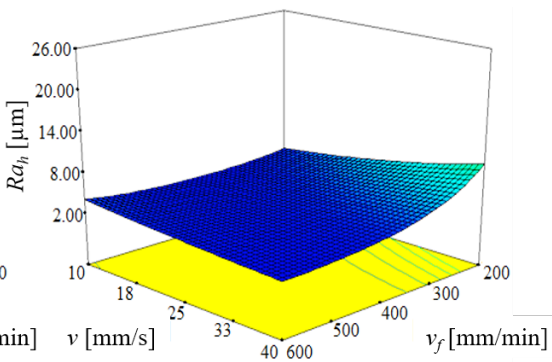

c)

Fig. 3. Influence of printing speed $v$ and feed speed of milling tool $v_{f}$ on surface roughness $R a_{h}$ by different layer heights $h$ using nozzle size $D_{2}=1.1 \mathrm{~mm}$; a) $h=0.3 \mathrm{~mm}$, b) $h=0.55 \mathrm{~mm}$, and c) $h=0.8 \mathrm{~mm}$ 
of the milling tool $v_{f}$. It can be concluded (see Figs. 2 and 3 ) that the minimal roughness $R a_{h}$ can be obtained through higher layers. That leads to fewer passages or gaps in between layers, which might be detected with the tip of the measuring device stylus. The models for both nozzles show that printing speed $v$ has only marginal effect on the surface roughness. However the best results (minimal roughness), especially with larger nozzle $D_{2}$, can be achieved, when maximal feed speed $v_{f}$ and printing speed are applied. Experimental observations have also revealed that otherwise, slow feeds in combination with high spindle speed of milling tool cause material overheating, which leads to winding of material on the tools' surface and therefore to poor surface quality.

\section{Regression models for roughness $R a_{1}$ :}

- $\quad$ Nozzle size $D_{1}=0.4 \mathrm{~mm}$ :

$$
\begin{aligned}
& R a_{l}=10.85149-3.71616 \cdot 10^{-4} \cdot n+7.16414 \cdot h- \\
& 0.082622 \cdot \phi-7.27924 \cdot 10^{-3} \cdot v_{f}+2.96111 \cdot a_{p}+ \\
& 5.80808 \cdot 10^{-4} \cdot n \cdot h+6.75758 \cdot 10^{-7} \cdot n \cdot v_{f}- \\
& 0.038625 \cdot h \cdot v_{f}+2.33778 \cdot 10^{-4} \cdot \phi \cdot v_{f}- \\
& 1.25556 \cdot 10^{-5} \cdot v_{f}^{2} .
\end{aligned}
$$

- $\quad$ Nozzle size $D_{2}=1.1 \mathrm{~mm}$ :

$R a_{l}=-28.06072+3.78833 \cdot 10^{-4} \cdot n+49.56052 \cdot h+$

$0.18399 \cdot \Phi-0.26487 \cdot v-7.98704 \cdot 10^{-3} \cdot v_{f}+$

$58.95257 \cdot a_{p}-7.85634 \cdot 10^{-6} \cdot n \cdot v-0.21452 \cdot h \cdot \Phi+$

$0.66534 \cdot v \cdot a_{p}-31.67556 \cdot h^{2}-66.1281 \cdot a_{p}^{2}$.

The conclusions obtained with previous regression models $\left(R a_{h}\right)$ can be applied also for the models of the surface roughness in the direction of the loading material $R a_{1}$. To achieve minimal roughness, the tool spindle speed $n$ must be set at level -1 , as shown in
Fig. 4. Higher spindle speed values cause material overheating and similar problems with material winding around tool, as found by interpretation of the feed speed $v_{f}$ in the perpendicular direction. The regression models also reveal that at the highest value of layer $h$, minimal surface roughness $R a_{1}$ can be achieved using minimal material compensation flow $\Phi$ (Figs. 5 and 6).

Regression models for roughness $R y_{l}$ :

- $\quad$ Nozzle size $D_{1}=0.4 \mathrm{~mm}$ :

$R y_{l}^{2.68}=71475.11377-3.97046 \cdot n+$

$86279.98635 \cdot h-613.28730 \cdot \phi+1212.98635 \cdot v-$

$158.1637 \cdot v_{f}+22158.41371 \cdot a_{p}+10.87269 \cdot n \cdot h+$

$5.37597 \cdot 10^{-3} \cdot n \cdot v_{f}-295.27319 \cdot h \cdot v_{f}$

$6.99385 \cdot \phi \cdot \mathrm{v}+2.15472 \cdot \phi \cdot \mathrm{v}_{f}-3.08891 \cdot 10^{-5} \cdot h^{2}-$

$8.9001 \cdot v^{2}$.

- $\quad$ Nozzle size $D_{2}=1.1 \mathrm{~mm}$ :

$R y_{l}=-129.23439-7.37746 \cdot 10^{-4} \cdot n+$

$265.11886 \cdot h+2.13655 \cdot \Phi-1.19240 \cdot v+$

$0.22008 \cdot v_{f}-3.26786 \cdot a_{p}+7.46030 \cdot 10^{-3} \cdot n \cdot h-$

$8.17239 \cdot 10^{-5} \cdot n \cdot v-1.26044 \cdot h \cdot \Phi-$

$2.44771 \cdot 10^{-3} \cdot \Phi \cdot v_{f}+4.41349 \cdot v \cdot a_{p}-$

$0.19900 \cdot v_{f} \cdot a_{p}-270.54667 \cdot h^{2}$.

The models in form of response surface diagrams presented in Figs. 7 and 8 give the same conclusions as in the case of the $R a$ roughness. The lowest spindle speed value has the lowest influence on heating of the workpiece material and its winding around the tool for both nozzle sizes. The higher feed speed $v_{f}$ leads to better roughness in both cases, while the milling depth $a_{p}$ has almost no influence on roughness $R y_{l}$ when

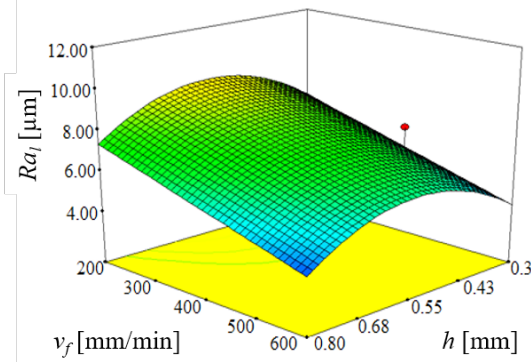

a)

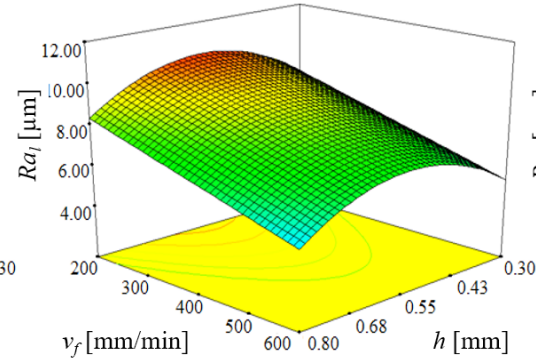

b)

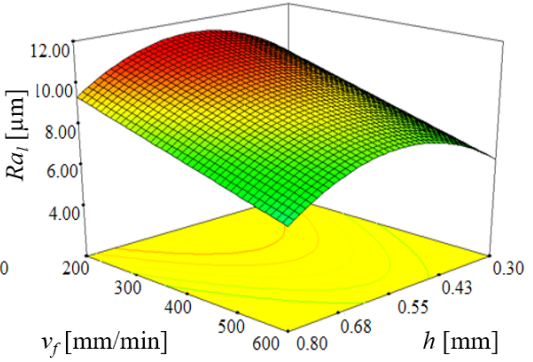

c)

Fig. 4. Influence of feed speed of milling tool $v_{f}$ and layer height $h$ on surface roughness $R a_{l}$ by different spindle speed of milling tool $n$ using nozzle size $D_{2}=1.1 \mathrm{~mm}, \Phi=70 \%, v=25 \mathrm{~mm} / \mathrm{s}, a_{p}=0.55 \mathrm{~mm}$; a) $n=10000 \mathrm{~min}^{-1}$, b) $n=15500 \mathrm{~min}^{-1}$, and c) $n=21000 \mathrm{~min}^{-1}$ 


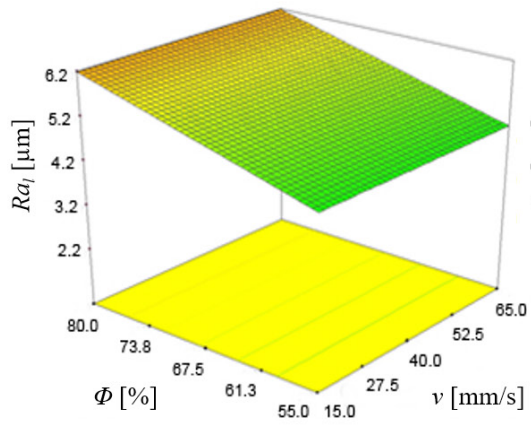

a)

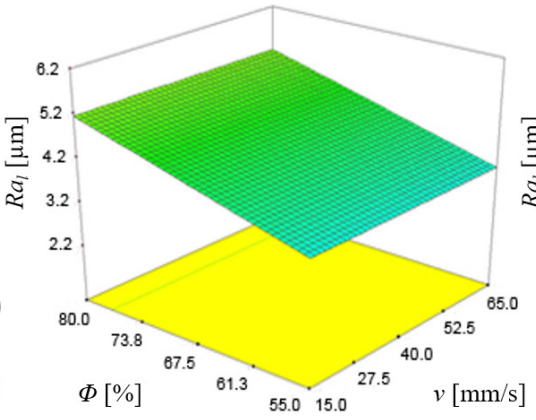

b)

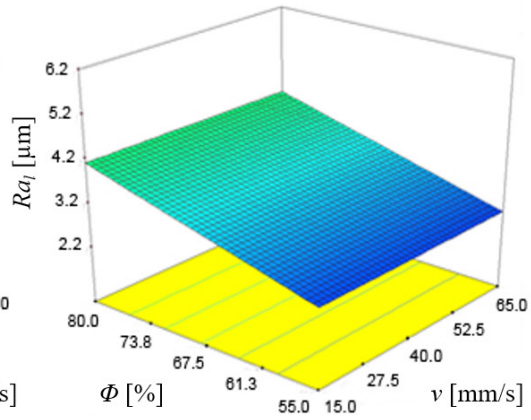

c)

Fig. 5. Influence of material compensation flow $\Phi$ and printing speed $v$ on surface roughness $R a_{l}$ by different layer heights $h$ using nozzle size $D_{1}=0.4 \mathrm{~mm}, n=10000 \mathrm{~min}^{-1}, v_{f}=600 \mathrm{~mm} / \mathrm{min}, a_{p}=0.1 \mathrm{~mm}$; a) $\left.h=0.1 \mathrm{~mm}, \mathrm{~b}\right) h=0.2 \mathrm{~mm}$, and c) $h=0.3 \mathrm{~mm}$

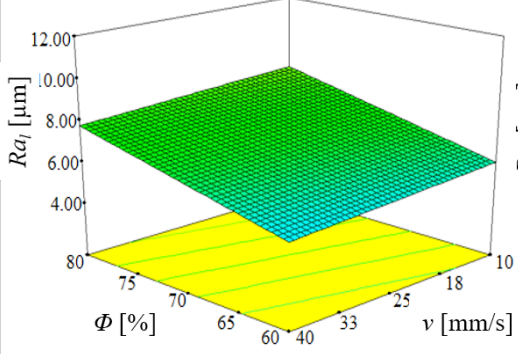

a)

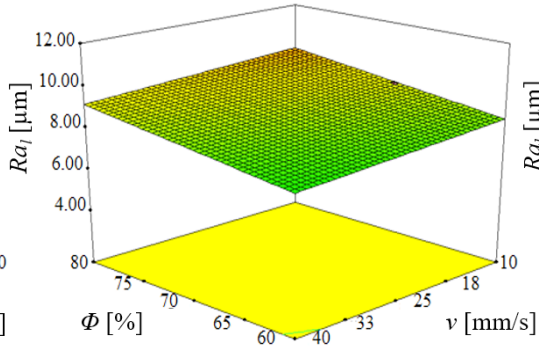

b)

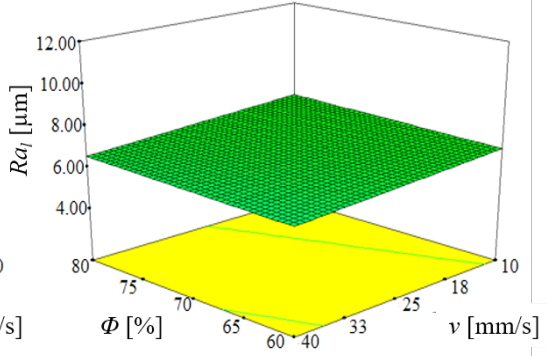

c)

Fig. 6. Influence of material compensation flow $\Phi$ and printing speed $v$ on surface roughness $R a_{l}$ by different layer heights $h$ using nozzle size $D_{2}=1.1 \mathrm{~mm}, n=15500 \mathrm{~min}^{-1}, v_{f}=400 \mathrm{~mm} / \mathrm{min}, a_{p}=0.55 \mathrm{~mm}$; a) $\left.h=0.3 \mathrm{~mm}, b\right) h=0.55 \mathrm{~mm}$, and c) $h=0.8 \mathrm{~mm}$

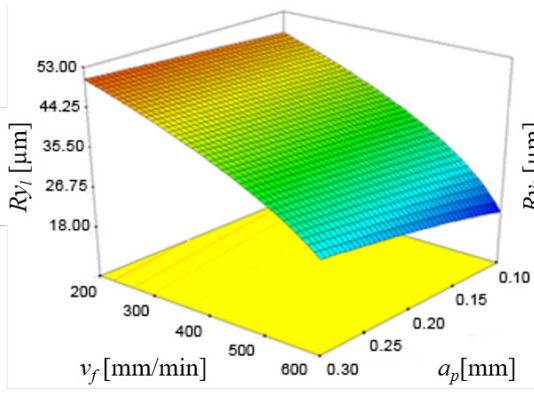

a)

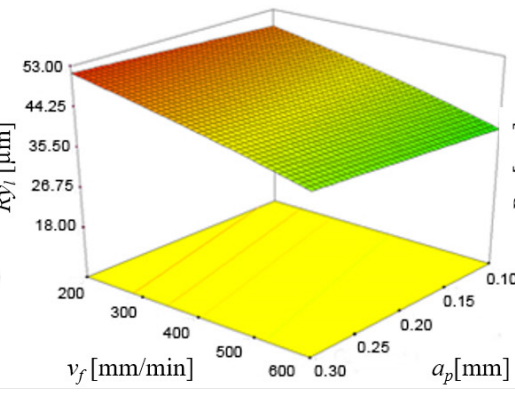

b)

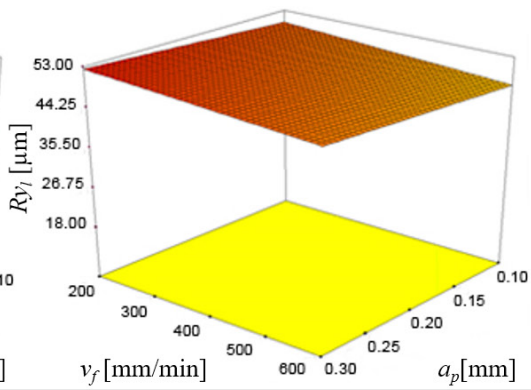

c)

Fig. 7. Influence of feed speed of milling tool $v_{f}$ and milling depth ap on surface roughness $R y_{l}$ by different spindle speed of milling tool $n$ using nozzle size $D_{1}=0.4 \mathrm{~mm}, h=0.3 \mathrm{~mm}, \Phi=55 \%, v=65 \mathrm{~mm} / \mathrm{s}$; a) $n=10000 \mathrm{~min}^{-1}$, b) $n=15500 \mathrm{~min}^{-1}$, and c) $n=21000 \mathrm{~min}^{-1}$

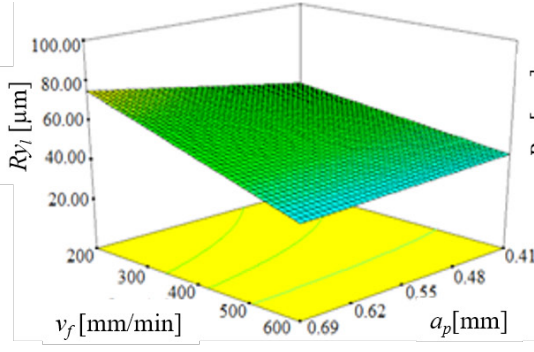

a)

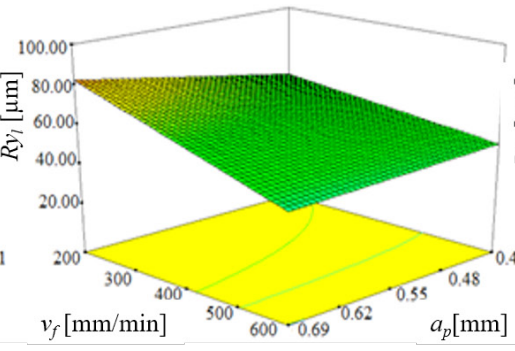

b)

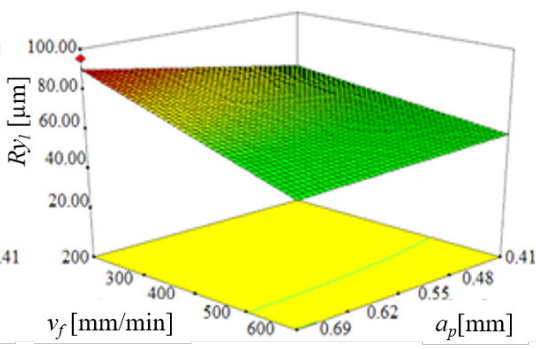

c)

Fig. 8. Influence of feed speed of milling tool $v$ and milling depth ap on surface roughness $R y_{l}$ by different spindle speed of milling tool $n$ using nozzle size $D_{1}=1.1 \mathrm{~mm}, h=0.55 \mathrm{~mm}, \Phi=70 \%, v=25 \mathrm{~mm} / \mathrm{s}$; a) $n=10000 \mathrm{~min}^{-1}, \mathrm{~b}$ ) $n=15500 \mathrm{~min}^{-1}$, and c) $n=21000 \mathrm{~min}-1$ 
the larger nozzle is applied. In the case of nozzle size $D_{1}=0.4 \mathrm{~mm}$, milling depth $a_{\mathrm{p}}$ is proportional to the roughness $R y_{l}$ (see Fig. 7). The finest roughness can be achieved by the use of minimal values of the milling depth $a_{p}$.

\section{Regression models for material deposition $M D$ :}

- $\quad$ Nozzle size $D_{1}=0.4 \mathrm{~mm}$ :

$$
\sqrt{M D}=0.54968+8.32623 \cdot 10^{-3} .
$$

- $\quad$ Nozzle size $D_{2}=1.1 \mathrm{~mm}$ :

$$
\begin{aligned}
& \ln (M D)=-0.53217-0.18252 \cdot h+ \\
& 0.027546 \cdot \Phi+2.39521 \cdot 10^{-4} \cdot h \cdot \Phi+ \\
& 0.11018 \cdot h^{2}-9.53869 \cdot 10^{-5} \cdot \Phi^{2} .
\end{aligned}
$$

As can be observed from $M D$ models in form of equations the main influence on material deposition $M D$ for both nozzle sizes has material compensation flow $\Phi$ with proportional effect. In addition, as seen from the response surface diagram for the nozzle size $D_{2}=1.1 \mathrm{~mm}$ (Fig. 9) if the minimal material flow is used the layer height $h$ has no big influence on the material deposition.

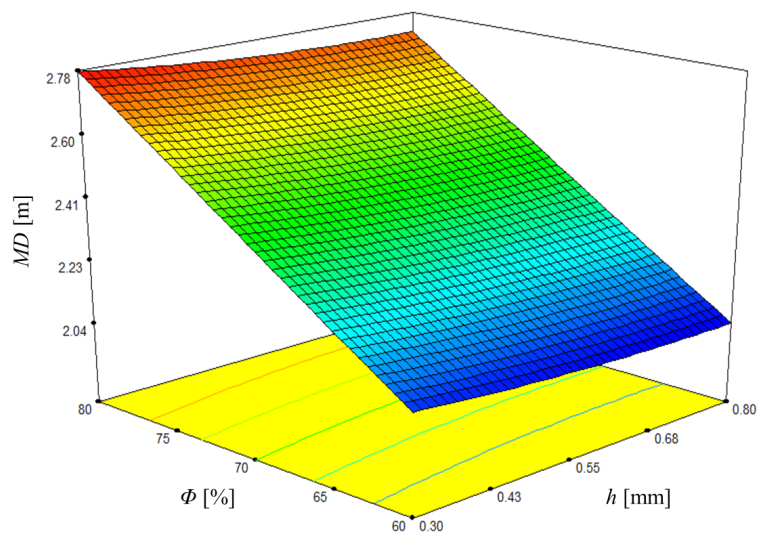

Fig. 9. Influence of layer height $h$ and material compensation flow $\Phi$ on material deposition $M D$ using nozzle size $D_{2}=1.1 \mathrm{~mm}$

Regression models for hybrid manufacturing time t:

- $\quad$ Nozzle size $D_{1}=0.4 \mathrm{~mm}$ :

$$
\begin{aligned}
& 1 / t=-1.488 \cdot 10^{-5}+1.2647 \cdot 10^{-3} \cdot h+ \\
& 5.61659 \cdot 10^{-7} \cdot v .
\end{aligned}
$$

- Nozzle size $D_{2}=1.1 \mathrm{~mm}$ :

$\frac{1}{\sqrt{t}}=0.011849+0.027023 \cdot h+9.22941 \cdot 10^{-5} \cdot v$.
The biggest influence on hybrid manufacturing time $t$ has a layer height $h$. Shorter production times and minimal surface roughness are achieved by higher layer implementations. Response surface diagrams for both nozzle sizes (Figs. 10 and 9) show that at maximal layer height $h$ printing speed $v$ has no big influence on the hybrid manufacturing time.

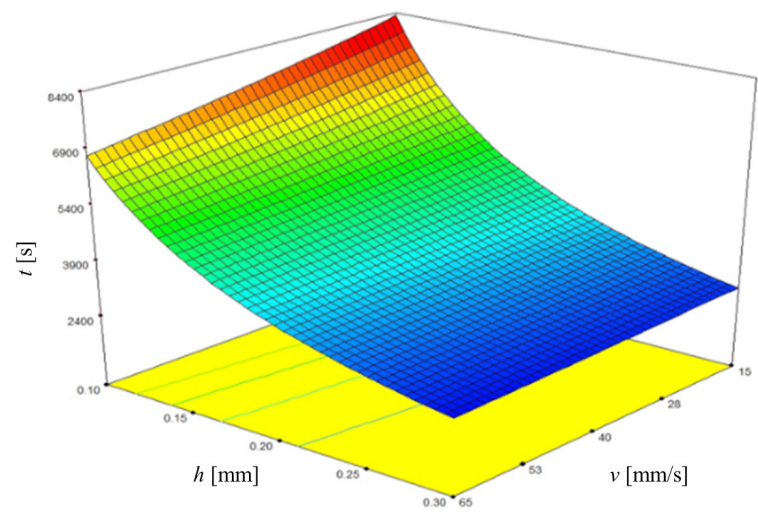

Fig. 10. Influence of layer height $h$ and printing speed $v$ on time for manufacturing $t$ for nozzle size $D_{1}=0.4 \mathrm{~mm}$

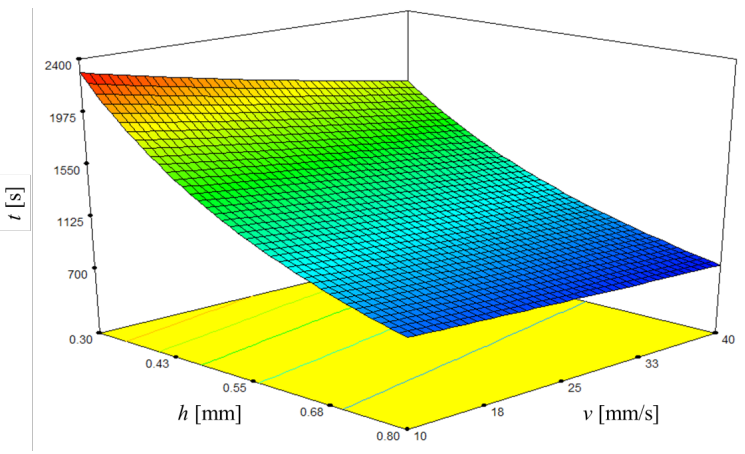

Fig. 11. Influence of layer height $h$ and printing speed $v$ on time for manufacturing $t$ for nozzle size $D_{2}=1.1 \mathrm{~mm}$

\subsection{Optimization of Parameters in the Hybrid Manufacturing}

Faster production, with lower production costs and improved product quality, is what it is usually striving for. Accordingly, obtained regression models presented in chapter 2.4, were further deployed for hybrid manufacturing optimization according to three equally-weighted criteria: the minimal surface roughness $R a$ and $R y$ (in both directions), minimal material deposition $M D$ and shortest production time $t$. The parameters settings for optimal technological solution for both nozzle sizes are shown in Table 8 .

The optimal technological parameters settings, presented in the Table 8 , match with the conclusions obtained during interpretation of the regression models. Nevertheless, the obtained optimal 
parameters were checked with the confirmation test (Tables 9 and 10). The results of the measured output parameters after the confirmation test fit well with the optimization predicted values for both nozzle sizes as shown in the Tables 9 and 10.

Table 8. Optimal technological parameters settings for both nozzle sizes

\begin{tabular}{lcc}
\hline & \multicolumn{2}{c}{ Nozzle size } \\
\cline { 2 - 3 } & $D_{1}=0.4 \mathrm{~mm}$ & $D_{2}=1.1 \mathrm{~mm}$ \\
\hline$n\left[\mathrm{~mm}^{-1}\right]$ & 10000 & 10000 \\
\hline$h[\mathrm{~mm}]$ & 0.3 & 0.8 \\
\hline$\Phi[\%]$ & 55 & 60 \\
\hline$v[\mathrm{~mm} / \mathrm{s}]$ & 65 & 40 \\
\hline$v_{f}[\mathrm{~mm} / \mathrm{min}]$ & 600 & 600 \\
\hline$a_{p}[\mathrm{~mm}]$ & 0.10 & 0.46 \\
\hline
\end{tabular}

Table 9. Results of confirmation test obtained with nozzle size $D_{1}=0.4 \mathrm{~mm}$

\begin{tabular}{lcc}
\hline & $\begin{array}{c}\text { With optimization } \\
\text { predicted values }\end{array}$ & $\begin{array}{c}\text { After confirmation } \\
\text { test measured values }\end{array}$ \\
\hline$R a_{h}[\mu \mathrm{m}]$ & 1.96 & 2.05 \\
\hline$R y_{h}[\mu \mathrm{m}]$ & Useless model & Not measured \\
\hline$R a_{l}[\mu \mathrm{m}]$ & 2.71 & 2.74 \\
\hline$R y_{l}[\mu \mathrm{m}]$ & 18.56 & 19.24 \\
\hline$M D[\mathrm{~m}]$ & 1.02 & 1.01 \\
\hline$t[\mathrm{~s}]$ & 2494 & 2496 \\
\hline
\end{tabular}

Table 10. Results of confirmation test obtained with nozzle size $D_{2}=1.1 \mathrm{~mm}$

\begin{tabular}{lcc}
\hline & $\begin{array}{c}\text { With optimization } \\
\text { predicted values }\end{array}$ & $\begin{array}{c}\text { After confirmation } \\
\text { test measured values }\end{array}$ \\
\hline$R a_{h}[\mu \mathrm{m}]$ & 2.18 & 2.16 \\
\hline$R y_{h}[\mu \mathrm{m}]$ & Useless model & Not measured \\
\hline$R a_{l}[\mu \mathrm{m}]$ & 2.69 & 2.60 \\
\hline$R y_{l}[\mu \mathrm{m}]$ & 18.05 & 18.18 \\
\hline$M D[\mathrm{~m}]$ & 2.04 & 2.04 \\
\hline$t[\mathrm{~s}]$ & 724 & 723 \\
\hline
\end{tabular}

Comparison of parameters settings for both nozzle sizes reveals that the same conclusions can be applied for both of them. For the achievement of optimal conditions according to required criteria, maximal layer height $h$, maximal printing speed $v$, minimal material compensation flow $\Phi$, minimal spindle speed of milling tool $n$, maximal feed speed of milling tool $v_{f}$, and milling depth $a_{p}$ at minimal level should be selected.

For the consideration of the minimal surface roughness, the size of the nozzle bears no importance.
Furthermore, a noticeable difference in the material consumption between the two nozzle sizes has minor importance due to the affordable material price. However, when the hybrid manufacturing time $t$ is important, then it is better to use bigger nozzle size $D_{2}=1.1 \mathrm{~mm}$ which allows for three times shorter manufacturing time.

\section{CONCLUSIONS}

A statistical approach has been applied to investigate the influence of the technological parameters on the surface roughness, material deposition and hybrid manufacturing time.

Based on the analysis of regression models, the optimal spindle speed of the milling tool $n$, layer height $h$, material compensation flow $\Phi$, printing speed $v$, feed speed of the milling tool $v_{f}$ and milling depth $a_{p}$ have been identified. Conclusions obtained with optimal results by both nozzle sizes can be interpreted in the same manner:

1. Surface roughness after hybrid manufacturing: the main influence on roughness has layer height $h$. With the highest layer, minimal roughness ( $R a$ and $R y$ ) can be achieved in both directions of measurement. At such layer height, material compensation flow $\Phi$ has no influence on roughness. The high spindle speed of the milling tool $n$ can cause material winding on the tools surface. Therefore, the optimal value for this parameter is selected as a minimal value. The feed speed of the milling tool $v_{f}$ affects inversely proportional the roughness. A smaller milling depth $a_{p}$ assures better roughness when the smaller nozzle is being applied, while in the case of larger nozzle the depth of milling has only marginal influence.

2. Material deposition in the hybrid manufacturing: the main influence on material deposition $M D$ has material compensation flow $\Phi$, which effects proportionally.

3. Hybrid manufacturing time: the shortest manufacturing time can be achieved and the highest surface quality can be obtained when both, the fastest printing and the highest layer, are applied.

Main conclusion: with the application of the bigger nozzle size $\left(D_{2}=1.1 \mathrm{~mm}\right)$ the same final surface roughness can be achieved as with smaller (standard, $D=0.4 \mathrm{~mm}$ ) nozzle size, but at three times higher productivity. 


\section{REFERENCES}

[1] Grguraš, D. (2015). Preparation of the CNC-Code and Optimization of the Technological Parameters in the Hybrid Manufacturing. MSc thesis, University of Ljubljana, Faculty of Mechanical Engineering, Ljubljana. (in Slovene)

[2] Kozan, Ž. (2016), Optimization of the Technological Parameters in Hybrid Manufacturing. BSc thesis, University of Ljubljana, Faculty of Mechanical Engineering, Ljubljana. (in Slovene)

[3] Song, Y.-A., Park, S., Choi, D., Jee, H. (2005). 3D welding and milling: Part I - a direct approach for freeform fabrication of metallic prototypes. International Journal of Machine Tools and Manufacture, vol. 45, no. 9, p. 1057-1062, D0l:10.1016/j. ijmachtools.2004.11.021.

[4] Song, Y.-A., Park, S., Choi, D., Chae, S.-W. (2005). 3D welding and milling: Part II - optimization of the 3D welding process using an experimental design approach. International Journal of Machine Tools and Manufacture, vol. 45, no. 9, p. 10631069, DOl:10.1016/j.ijmachtools.2004.11.022.

[5] Boivie, K., Homar, D., Dolinšek, S., Broetan V. (2012). The hybrid manufacuring cell: an integrated solution for additive manufacturing with CNC machining. $4^{\text {th }}$ International Conference on Additive Technologies, Maribor, p. 1-15.

[6] Yamazaki, T. (2016). Development of a Hybrid Multitasking Machine Tool: Integration of Additive Manufacturing Technology with CNC Machining. Procedia CIRP, vol. 42, p. 8186, D0I:10.1016/j.procir.2016.02.193.

[7] Lee, W.-C., Wei, C.-C., Chung, S.-C. (2014). Development of a hybrid rapid prototyping system using low-cost fused deposition modeling and five-axis machining. Journal of Materials Processing Technology, vol. 214, no. 11, p. 23662374, D0I:10.1016/J.jmatprotec.2014.05.004.

[8] Mohamed, O.A., Masood, S.H., Bhowmik, J.L. (2016). Optimization of fused deposition modeling process parameters for dimensional accuracy using l-optimality criterion. Measurement, vol. 81, p. 174-196, D0I:10.1016/j. measurement.2015.12.011.

[9] Kaveh, M., Badrossamay, M., Foroozmehr, E., Etefagh, A.H. (2015). Optimization of the printing parameters affecting dimensional accuracy and internal cavity for HIPS material used in fused deposition modeling processes. Journal of Materials Processing Technology, vol. 226, p. 280-286, D0l:10.1016/j.jmatprotec.2015.07.012.

[10] Rao, R.V., Rai, D.P. (2016). Optimization of fused deposition modeling process using teaching-learning-based optimization algorithm. Engineering Science and Technology, an International Journal, vol. 19, no. 1, p. 587-603, D0l:10.1016/j.jestch.2015.09.008.

[11] Hassine H., Barkallah M., Bellacicco A., Louati J., Riviere A., Haddar M. (2015). Multi Objective Optimization for Sustainable Manufacturing, Application in Turning. International Journal of Simulation Modelling, vol. 14, no. 1, p. 98-109, D0l:10.2507/ IJSIMM14(1)9.292.

[12] Barkallah M., Louati J., Haddar M. (2012). Evaluation of Manufacturing Tolerance Using a Statistical Method and Experimentation. International Journal of Simulation Modelling, vol. 11, no. 1, p. 5-16, D0l:10.2507/ IJSIMM11(1)1.194. 